\title{
Phase randomness in a one-dimensional disordered absorbing medium
}

\author{
V. Freilikher and M. Pustilnik* \\ The Jack and Pearl Resnick Institute of Advanced Technology, \\ Department of Physics, Bar-Ilan University, Ramat-Gan 52900, Israel
}

(March 31, 2018)

\begin{abstract}
Analytical study of the distribution of phase of the transmission coefficient through 1D disordered absorbing system is presented. The phase is shown to obey approximately Gaussian distribution. An explicit expression for the variance is obtained, which shows that absorption suppresses the fluctuations of the phase. The applicability of the random phase approximation is discussed.
\end{abstract}

PACS numbers: 42.25.Bs, 72.15.Rn, 05.40.+j, 78.20.Ci 
Rigorous results for the reflection of waves from a disordered absorbing one-dimensional medium have been obtained a long time ago [1]. Nevertheless, despite its evident significance, the problem of wave propagation in absorbing disordered media has received less attention as compared to the studies of waves in passive (nonabsorptive) systems. In the pioneering work [2] the possibility of the localization of light has been predicted, which gave a new boost to the studies of classical waves in random media. It was believed during the years that the effect of absorption on the classical wave transport is similar to that of inelastic scattering processes on electrons in disordered solids. Indeed, it is well- known that when the incoherent effects are modelled by introducing an optical (imaginary) potential, the theory simplifies drastically [3]. Only recently it was understood that the role of absorption differs fundamentally from the role of inelastic scattering for electrons [4] (see also discussion in [5], [6]). The source of this difference is that absorption corresponds to the actual removal of particles, but does not affect the phase coherence. Conversely, the inelastic scattering causes the loss of the phase memory and suppresses the interference effects, but preserves the number of particles. Recently there have been a number of works dealing with the effect of absorption (and related problem of the coherent amplification) on the wave transport in random media [4]- [12].

We study in this paper the probability distribution of the phase $\theta$ of the transmission coefficient $t$ through $1 \mathrm{D}$ random absorbing media. It is well-established now that in a passive (nonabsorbing) 1D medium the interference leads to the strong localization of all eigenstates in the presence of arbitrary weak disorder. If the length of the scattering region $L$ is large compared to the characteristic length of elastic scattering $l$, the transmittance $T=|t|^{2}$ decreases exponentially with the length in average. On the other hand, $T$ strongly fluctuates and obeys the log-normal distribution. If the disorder is weak, the phases of the reflection and transmission coefficients become random [13], [14]. One may assume that the phase $\varphi$ of the reflection coefficient is distributed uniformly in the interval $(0,2 \pi)$ and independently on the amplitude of the reflection coefficient and amplitude and phase of the transmission coefficient. This assumption will be referred below as the random phase 
approximation (RPA). RPA, however, does not implies that the phase $\theta$ of the transmission coefficient is also uniformly distributed. Indeed, the recently obtained formula reads [15]:

$$
\left\langle t / t^{*}\right\rangle=\left\langle e^{2 i \theta}\right\rangle=(1+L / l) e^{-3 L / l} .
$$

Note that calculating this average with the uniform distribution for $\theta$ one would obtain zero.

The arguments presented above suggest that RPA can be used for calculation of the distribution of the absolute phase $\theta \in(-\infty, \infty)$. When the absorption is zero, $\theta$ relates to the density of states in the scatterer by $\rho=\pi^{-1}[\partial(k L+\theta) / \partial E], E=k^{2}$ [16]. Therefore $(k+\theta / L) / \pi$ is the total number of states per unit length, which is self-averaged in the limit $L / l \gg 1$ [17], and obeys almost Gaussian distribution with $\operatorname{var}(\theta / L) \propto L^{-1}$. The natural question arises in the context of the discussion above is how absorption affects this distribution. This question is addressed below.

A wave $U(z)$ with the wavenumber $k$ propagating in a one dimensional random medium satisfies the equation

$$
\left(\frac{d^{2}}{d z^{2}}+k^{2} \epsilon(z)\right) U(z)=0,
$$

where the dielectric permittivity $\epsilon(z)$ is equal to 1 for $z<0$ and $z>L$ and $\epsilon(z)=1+\delta \epsilon(z)$ for $0 \leq z \leq L$. We assume for simplicity that the imaginary part of $\delta \epsilon(z)$, which is responsible for the absorption, is constant, while the real part $\varepsilon(z)$ is a random function of $z$, which may be approximated by Gaussian white noise:

$$
\begin{gathered}
\delta \epsilon(z)=\varepsilon(z)+i / k l_{a}, \\
\langle\varepsilon(z)\rangle=0, \quad\left\langle\varepsilon(z) \varepsilon\left(z^{\prime}\right)\right\rangle=\frac{4}{k^{2} l} \delta\left(z-z^{\prime}\right) .
\end{gathered}
$$

Here the length of elastic scattering $l$ in a system without absorption and the length of absorption $l_{a}$ in a system without disorder have been introduced. The reflection $(r)$ and transmission $(t)$ coefficients are defined according to the wave behavior outside the disordered region: 


$$
U \propto \begin{cases}e^{-i k z}+r e^{i k z}, & z \geq L \\ t e^{-i k z}, & z \leq 0\end{cases}
$$

To proceed, we introduce the joint distribution function

$$
\mathcal{P}_{L}(x, \theta)=\langle\delta(x-x(L)) \delta(\theta-\theta(L))\rangle,
$$

where we have used the parametrization $r=\tanh (x / 2) e^{i \varphi}, t=|t| e^{i \theta},-\infty<\theta<\infty$. To derive the Fokker-Planck equation for $\mathcal{P}_{L}(x, \theta)$ it is convenient to use the so-called invariant imbedding equations for $r$ and $t$ (a detailed description of the similar calculation can be found for instance in [7], [18]). Assuming that RPA is applicable for length $L$ larger than the wavelength $(k L \gg 1)$ and in the limit of weak scattering $(k l \gg 1)$ and weak absorption $\left(k l_{a} \gg 1\right)$, we obtain the equation

$$
\frac{\partial}{\partial S} \mathcal{P}_{S}(x, \theta)=\left(\frac{\partial}{\partial x}\left(\frac{\partial}{\partial x}+\frac{d \Omega_{\beta}}{d x}\right)+F(x) \frac{\partial^{2}}{\partial \theta^{2}}\right) \mathcal{P}_{S}(x, \theta),
$$

where

$$
\begin{gathered}
\Omega_{\beta}(x)=-\ln (\sinh x)+\beta \cosh x, \\
F(x)=\frac{1}{4}\left(3-\frac{1}{\cosh ^{2} x / 2}\right)=\frac{1}{4}\left(2+R_{S}\right) .
\end{gathered}
$$

Here $S=L / l$ and $l$ is the scattering length for zero absorption. The parameter $\beta=l / l_{a}$ describes the relative strength of absorption. Alternatively, Eq.(6) can be derived by the method of Ref. [15]. Indeed, for $\beta=0$ Eq.(6) is equivalent to the equation for expectation values of Ref. [19]. It should be noticed that it is not necessary to assume the white noise distribution for $\varepsilon(z)$ to derive Eq.(河). It is enough to require the weakness of scattering $k l \gg 1$ [15].

After integration over $\theta$ Eq. (6) reduces to the well-known equation for the probability density distribution of $x$ [1]. The solution of the later equation saturates at $S \gg 4$ to the limiting $S$-independent distribution [1], [7], [8]

$$
\mathcal{P}_{\infty}(x)=\beta e^{\beta-\Omega_{\beta}(x)}
$$


with $\Omega_{\beta}$ given by Eq. (7). The distribution (8) implies the following limiting value for the average reflectance [1]:

$$
R_{\infty}=\lim _{S \rightarrow \infty}\left\langle R_{S}\right\rangle=1+2 \beta e^{2 \beta} \operatorname{Ei}(-2 \beta) \simeq \begin{cases}1-2 \beta \ln (1 / \beta), & \beta \ll 1 \\ (2 \beta)^{-1}, & \beta \gg 1\end{cases}
$$

where $\operatorname{Ei}(x)=\int_{-\infty}^{x} d y e^{y} / y$.

Here is a point to discuss the validity of RPA, which was essentiall for derivation of Eq.(6), and therefore underlies the result of Eq.(9) that predicts a monotonic decrese of $R_{\infty}$ with $\beta$. Despite this result being known for more than twenty years, it was only recently understood [5], [6] that this prediction becomes wrong for large $\beta$. Moreover, it has been verified numerically that RPA fails at the same values of $\beta[6]$. Let us estimate now the range of validity of RPA. In the limit $\beta=l / l_{a} \gg 1$ the wave penetrates into disordered absorbing medium at a distance which is much smaller than elastic scattering length $l$. This means that disorder may lead only to a small correction to $R_{\infty}$, which can be taken into account by means of perturbation expansion in the small parameter $1 / \beta$ :

$$
R_{\infty}=R^{0}+R^{1} \beta^{-1}+O\left(\beta^{-2}\right) .
$$

The zeroth-order term here does not depend on $\beta$ but on the parameter $k l_{a}$ only. In the limit $k l_{a} \ll 1$ (strong absorption, which was the case in [5]) this term is equal to 1 . This is easy to understand since this limit corresponds to a large conductivity, where the skin-effect results in the almost perfect reflection. However, we are considering here the opposite limit of the weak absorption $\left(k l_{a} \gg 1\right)$. In this limit, $\beta \gg 1$ corresponds to the absence of the elastic scattering rather than to the strong absorption, and $R^{0}$ is nothing but the reflectance of the ideal (ordered) lossy system. Calculating the reflectance $R_{\infty}$ in the lowest order on $\left(k l_{a}\right)^{-1}$ and $\beta^{-1}$, we find that Eq.(10) takes the form

$$
R_{\infty} \simeq\left(4 k l_{a}\right)^{-2}+(2 \beta)^{-1}[1+O(1 / k l)]
$$

Eq.(III) is exact for $k l_{a} \gg 1$ and $k l \gg 1$. The first term in the r.h.s. in Eq.(II) is the reflectance of the semi-infinite clean media with the wave number $k_{1}=\left[k^{2}\left(1+i / k l_{a}\right)\right]^{1 / 2} \simeq$ 
$k\left(1+i / 2 k l_{a}\right)$ and is absent in Eq.(9). The second term represents the correction due to disorder and for $\beta \gg 1$ coincides exactly with Eq.(9). Since RPA was the only approximation made in derivation of the non-perturbative result Eq.(9), one immediately finds that RPA is applicable if, in addition to the weakness of both the scattering and absorption, the following inequality holds: $\left(4 k l_{a}\right)^{-2} \ll(2 \beta)^{-1}$, or

$$
\beta \ll \beta_{0} \sim 8\left(k l_{a}\right)^{2}
$$

These simple arguments are consistent with the recent numerical simulations [6], which clearly show that RPA is valid only when $\beta$ is smaller than some $\bar{\beta}(\beta \ll \bar{\beta})$, for which $R_{\infty}(\beta)$ takes its minimum. Eq. (12) provides therefore the value of $\bar{\beta} \sim \beta_{0}$ in the leading order in the parameter $1 / k l$, characterizing the weakness of disorder.

Now that we have established the limits of the validity of Eq.(6) in Eq.(12), let us turn to its solution. It is natural to assume that correlations between the phase $\theta$ and reflectance $R$ are negligible, and distribution (5) factorizes (see e.g. [20]):

$$
\mathcal{P}_{S}(x, \theta) \simeq P_{S}(x) P_{S}(\theta), S \gg 1
$$

Integrating Eq. (6) by making use of this conjecture, we get

$$
\frac{\partial}{\partial S} \mathcal{P}_{S}(\theta) \simeq \frac{1}{4}\left(2+\left\langle R_{S}\right\rangle\right) \frac{\partial^{2}}{\partial \theta^{2}} \mathcal{P}_{S}(\theta),
$$

which immediately results in the Gaussian distribution with zero average $\langle\theta\rangle=0$ and variance

$$
\operatorname{var} \theta=\left(1+R_{\infty} / 2\right) S
$$

One can see from Eqs.(9),(15) that the absorption suppresses fluctuations of $\theta$. Combining Eq.(15) with the recently obtained result $\operatorname{var}(\ln T)=2 R_{\infty} S$ [7] we obtain another useful formula

$$
\operatorname{var} \theta=S+\frac{1}{4} \operatorname{var}(\ln T)
$$


Calculating $\left\langle e^{2 i \theta}\right\rangle$ for $\beta=0$ with the Gaussian distribution specified by (15) one recovers Eq.(11) with an exponential accuracy.

It is interesting to note that Eq.(15) is rather general, and can be obtained without the conjecture (13). To do this we use the formula

$$
\operatorname{var} \theta=-\left(\frac{d^{2}\left\langle e^{i p \theta}\right\rangle}{d\left(p^{2}\right)}\right)_{p=0}
$$

To calculate the characteristic function $\left\langle e^{i p \theta}\right\rangle$ we consider the Fourier-transform of (6)

$$
\frac{\partial}{\partial S} P_{S}(x ; p)=\left(\frac{\partial}{\partial x}\left(\frac{\partial}{\partial x}+\frac{d \Omega_{\beta}}{d x}\right)-p^{2} F(x)\right) P_{S}(x ; p)
$$

Integrated over $x, P_{S}(x ; p)$ gives us a characteristic function $\left\langle e^{i p \theta}\right\rangle$. Following the procedure used in [7] we substitute

$$
P_{S}(x ; p)=e^{-\Omega_{\beta} / 2} \Psi(x ; S)
$$

which yields an imaginary time Scrödinger equation for $\Psi$. Solution of this equation in spectral representation takes the form

$$
\Psi(x ; S)=\sum_{n} e^{-E_{n} S} \psi_{n}(x)\left[\psi_{n}(y) e^{\Omega_{\beta}(y) / 2}\right]_{y \rightarrow 0}
$$

where $E_{n}$ and $\psi_{n}$ are eigenvalues and real normalized eigenfunctions of the Scrödinger operator

$$
\left(-\frac{d^{2}}{d x^{2}}+V_{p}(x)\right) \psi_{n}(x)=E_{n} \psi_{n}(x)
$$

with potential

$$
V_{p}(x)=\frac{1}{4}\left(1-\sinh ^{-2} x\right)+\frac{p^{2}}{4}\left(3-\cosh ^{-2} x / 2\right)-\beta \cosh x+\frac{\beta^{2}}{4} \sinh ^{2} x
$$

It can be easily seen [0] that the potential $V_{p}(x)$ has a minimum at $x \sim \ln (4 / \beta)$, and the large $S$ behavior of $P_{S}(x ; p)$ is dominated by the energy level localized in this well. For $p=0$ the energy of this level is exactly 0 and the corresponding wave function is 
$\psi_{0}^{0}=\left(\mathcal{P}_{\infty}(x)\right)^{1 / 2}=\beta^{1 / 2} e^{\beta / 2-\Omega_{\beta}(x)}$. For small $p^{2}$ (but for arbitrary $\beta$ ) the perturbation theory yields:

$$
E_{0}(p)=\left\langle\psi_{0}^{0}\left|p^{2} F(x)\right| \psi_{0}^{0}\right\rangle+o\left(p^{2}\right)=\frac{1}{4} p^{2}\left(2+R_{\infty}\right)+o\left(p^{2}\right)
$$

Therefore for $S \gg 4$

$$
\left\langle e^{i p \theta}\right\rangle \propto e^{-E_{0} S}=\exp \left[-\frac{1}{4} p^{2}\left(2+R_{\infty}\right)+o\left(p^{2}\right)\right]
$$

Making use of Eq. (17), we end up with Eq. (15).

To conclude, we have studied analytically the influence of small absorption on the distribution of the phases of the reflection and transmission coefficients through 1D weakly disordered random media. It was shown that the phase $\varphi$ of the reflection coefficient can be treated as a uniformly distributed when the absorption is weak enough to satisfy the inequality Eq.(12). An explicit expression for the variance of the phase $\theta$ of the transmission coefficient was obtained (Eq.(15)). The result shows that the presence of the absorption slows down the evolution of $\theta$ towards a random limit.

We thank I. Yurkevich for helpful discussions.

* present address: Department of Physics, University of Illinois at Chicago, Chicago, IL 60607

[1] W. Kohler and G. Papanicolaou, SIAM J. Appl. Math. 30, 263 (1976).

[2] S. John, Phys. Rev. Lett. 53, 2169 (1984).

[3] A. D. Stone and P. A. Lee, Phys. Rev. Lett. 54, 1196 (1985).

[4] R. L. Weaver, Phys. Rev. B 47, 1077 (1993); M. Yosefin, Europhys. Lett. 25, 675 (1994); M. Kaveh and E. Kogan, in Photonic Band Gaps and Localization, ed. by C. Soukoulis (Plenum Press, New York, 1993), p. 187.

[5] A. Rubio and N. Kumar, Phys. Rev. B 47, 2420 (1993). 
[6] A. K. Gupta and A. M. Jayannavar, Phys. Rev. B 52, 4156 (1995).

[7] V. Freilikher, M. Pustilnik, and I. Yurkevich, Phys. Rev. Lett. 73, 810 (1994); Phys. Rev. B 50, 6017 (1994).

[8] P. Pradhan and N. Kumar, Phys. Rev. B 50, 9644 (1994).

[9] A. Yu. Zyuzin, Phys. Rev. E 51, 5274 (1995).

[10] C. W. J. Beenakker, J. C. J. Paasschens, and P. W. Brouwer, preprint (cond-mat/9601024); T. Sh. Misirpashaev and C. W. J. Beenakker, preprint (cond-mat/9607119).

[11] J. C. J. Paasschens, T. Sh. Misirpashaev and C. W. J. Beenakker, preprint condmat/9602048); T. Sh. Misirpashaev, J. C. J. Paasschens and C. W. J. Beenakker, preprint (cond-mat/9607118).

[12] V. Freilikher, M. Pustilnik, and I. Yurkevich, preprint cond-mat/9605090).

[13] A. D. Stone, D. C. Allan and J. D. Joannopoulos, Phys. Rev. B 27, 836 (1983).

[14] J. B. Pendry, Adv. in Phys. 43, 461 (1994), P. D. Kirkman and J. B. Pendry, J. Phys. C 17, 4327 (1984).

[15] P. A. Mello and S. Tomsovic, Phys. Rev. Lett. 67, 342 (1991).

[16] D. J. Thouless, J. Phys. C 5, 77 (1972); P. W. Anderson and P. A. Lee, Progr. Theor. Phys. Suppl. 69, 212 (1980).

[17] I. M. Lifshits, S. A. Gredeskul and L. A. Pastur, Introduction to the Theory of Disordered Systems (Wiley, New York, 1988).

[18] R. Rammal and B. Doucot, J. Physique 48, 509 (1987).

[19] P. A. Mello, Phys. Rev. B 47, 16358 (1993).

[20] P. A. Mello, Phys. Rev. B 35, 1082 (1987). 